\title{
Le neoplasie associate a feocromocitoma/paraganglioma in quadri SDHx positivi o negativi: adenomi ipofisari, tumori stromali gastro-intestinali e tumori renali
}

\author{
Chiara Lopez $^{1}$ - Martina Bollati ${ }^{1}$. Mirko Parasiliti-Caprino ${ }^{1}$. Nunzia Prencipe ${ }^{1}$ - Alessandro Maria Berton ${ }^{1}$. \\ Ezio Ghigo ${ }^{1} \cdot$ Silvia Grottoli ${ }^{1} \cdot$ Mauro Maccario $^{1}$
}

Accettato: 22 dicembre 2020 / Pubblicato online: 11 agosto 2021

(c) The Author(s) 2021

Sommario I feocromocitomi e paragangliomi (PPGL) sono geneticamente determinati in almeno il $30 \%$ dei casi. Le mutazioni identificate più recentemente, in particolare quelle dei geni SDHx, possono favorire, seppur raramente, anche l'insorgenza di tumori stromali gastro-intestinali, carcinomi renali e adenomi ipofisari. Pertanto, in caso di diagnosi di una delle suddette neoplasie, il clinico dovrebbe valutare l'anamnesi personale e familiare alla ricerca di eventuali PPGL, così come in pazienti con PPGL associato a mutazione di SDHx, TMEM127 e MAX si dovrebbe indagare la presenza di neoplasie potenzialmente correlate.

Parole chiave Genetica $\cdot$ SDH .

Feocromocitoma/paraganglioma - Adenoma ipofisario ·

Carcinoma renale a cellule chiare . Tumori stromali

gastro-intestinali

\section{Introduzione}

Il feocromocitoma (PCC) e il paraganglioma (PGL) sono rare neoplasie neuroendocrine originate dal tessuto cromaffine della midollare del surrene o dei paragangli extrasurrenalici del sistema nervoso simpatico e parasimpatico.

Proposto da Silvia Grottoli.

Informazioni Supplementari La versione online contiene materiale supplementare disponibile su

https://doi.org/10.1007/s40619-021-00928-y.

$凶$ M. Parasiliti-Caprino

mirko.parasiliticaprino@unito.it

1 S.C. Endocrinologia, Diabetologia e Metabolismo U., A.O.U. Città della Salute e della Scienza di Torino, Dipartimento di Scienze Mediche, Università degli Studi di Torino, Torino, Italia
Storicamente, circa il $10 \%$ di questi tumori era ritenuto associato a una forma ereditaria (Sindrome da Neoplasie Endocrine Multiple di tipo 2, MEN2, Sindrome di von HippelLindau, VHL, e Neurofibromatosi di tipo 1, NF1); tuttavia, con la progressiva identificazione di nuove mutazioni geniche di suscettibilità (SDHA, SDHB, SDHC, SDHD, SDHAF2, TMEM127, MAX, FH, MDH2), si ritiene che almeno il $30 \%$ di essi sia causato da una mutazione germinale e possa quindi essere considerato ereditario [1, 2]. Pertanto, in accordo con le linee guida correnti, tutti i pazienti con diagnosi di feocromocitoma e paraganglioma (PPGL) dovrebbero essere sottoposti a counseling genetico per valutare l'indicazione a effettuare l'analisi genetica [3, 4].

Le suddette mutazioni germinali correlate all'insorgenza di PPGL predispongono allo sviluppo di altre neoplasie, differenti a seconda della specifica sindrome genetica [4]. In particolare, per le Sindromi del Paraganglioma Familiare (SDHx) sono state descritte associazioni con tumori stromali gastro-intestinali (Gastro-Intestinal Stromal Tumor, GIST), carcinomi a cellule renali (Renal Cell Carcinoma, RCC), adenomi ipofisari (Pituitary gland Tumor, PIT). In un'esigua percentuale di pazienti con mutazione SDHD, è stata evidenziata una correlazione con i carcinomi papilliferi tiroidei. La variante mutata del gene TMEM127 è stata invece riscontrata in una piccola percentuale di soggetti con RCC, così come la mutazione del gene MAX è stata riportata in pazienti con oncocitoma renale (Tabella 1) $[2,5,6]$.

\section{Le basi genetiche}

Le sindromi del Paraganglioma Familiare correlate a mutazioni dei geni SDHx vengono trasmesse con modalità autosomica dominante a penetranza incompleta. Più in dettaglio, i geni SDHD e SDHAF2 presentano imprinting mater- 
Tabella 1 Principali caratteristiche delle mutazioni a carico dei geni SDHx, TMEM127 e MAX e neoplasie associate [2]. AD, autosomico dominante; $C D T$, tumore differenziato tiroideo; GIST, tumore

\begin{tabular}{|c|c|c|c|c|c|c|c|c|}
\hline Gene & Ereditarietà & $\begin{array}{l}\text { Frequenza di } \\
\text { tutti i PPGL }\end{array}$ & $\begin{array}{l}\text { Penetranza } \\
\text { a } 70 \text { anni }\end{array}$ & $\begin{array}{l}\text { PGL } \\
\text { testa/collo }\end{array}$ & PCC & $\begin{array}{l}\text { PGL toracico- } \\
\text { addominali }\end{array}$ & $\begin{array}{l}\text { Rischio di } \\
\text { malignità }\end{array}$ & Tumori associati \\
\hline SDHA & $\mathrm{AD}$ & $<1 \%$ & $10 \%$ & $70 \%$ & $13 \%$ & $17 \%$ & basso & GIST PIT \\
\hline SDHB & $\mathrm{AD}$ & $8-10 \%$ & $30-40 \%$ & $20-30 \%$ & $20-25 \%$ & $50 \%$ & fino al $33 \%$ & RCC GIST PIT \\
\hline SDHC & $\mathrm{AD}$ & $2 \%$ & $25 \%$ a 60 anni & $77 \%$ & $3 \%$ & $17 \%$ & $4 \%$ & RCC GIST \\
\hline SDHD & $\begin{array}{l}\mathrm{AD} \text { con } \\
\text { imprinting } \\
\text { materno }\end{array}$ & $6 \%$ & $\begin{array}{l}80 \%(\mathrm{se} \\
\text { ereditato dal } \\
\text { padre) }\end{array}$ & $80 \%$ & $\begin{array}{l}\text { raro, ma } \\
\text { possibile }\end{array}$ & rari & $3-4 \%$ & RCC GIST PIT CDT? \\
\hline SDHAF2 & $\begin{array}{l}\mathrm{AD} \text { con } \\
\text { imprinting } \\
\text { materno }\end{array}$ & $1 \%$ & $\begin{array}{l}100 \% \text { a } 50 \text { anni } \\
\text { (se ereditato } \\
\text { dal padre) }\end{array}$ & $\begin{array}{l}\text { quasi la } \\
\text { totalità }\end{array}$ & raro & rari & non noto, basso? & non noto \\
\hline TMEM127 & $\mathrm{AD}$ & $0,6-2 \%$ & $\begin{array}{l}\text { non nota, in } \\
\text { una famiglia } \\
32 \% \text { a } 65 \text { anni }\end{array}$ & rari & $>95 \%$ & rari & $<5 \%$ & $\mathrm{RCC}$ \\
\hline MAX & $\mathrm{AD}$ & $1,1 \%$ & non nota & rari & $86 \%$ & $14 \%$ & dal 14 al $28 \% ?$ & $\mathrm{RCC}$ \\
\hline
\end{tabular}

stromale gastro-intestinale; $P C C$, feocromocitoma; $P G L$, paraganglioma; PIT, adenoma ipofisario; PPGL, feocromocitoma/paraganglioma; $R C C$, carcinoma a cellule renali

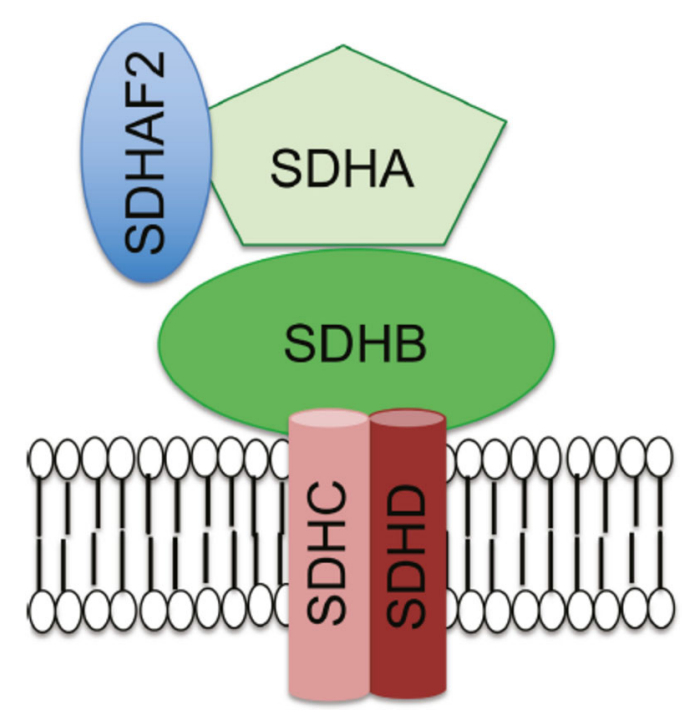

Fig. 1 Complesso della succinato deidrogenasi. SDHA e SDHB sono le subunità catalitiche del complesso a livello del citoplasma e contengono un sito di legame covalente per flavina adenina dinucleotide (FAD). SDHC e SDHD sono molecole idrofobiche che consentono l'ancoraggio del complesso alla membrana mitocondriale interna. Infine, vi sono 4 proteine addizionali (codificate dai geni SDHAF1-4), responsabili dell' assemblaggio del complesso (per semplicità in figura è mostrata solo SDHAF2) [2]

no; pertanto, si manifestano clinicamente soltanto se l'allele mutato viene trasmesso dal padre.

I geni SDHx codificano per le proteine del complesso II della succinato deidrogenasi a livello della catena di trasporto degli elettroni mitocondriale (Fig. 1). Il suddetto complesso catalizza l'ossidazione del succinato in fumarato nel contesto del ciclo degli acidi tricarbossilici, con il conseguente trasferimento di elettroni dal succinato all'ubiquinone (Fig. 1). Tali geni sono considerati oncosoppressori e lo- ro mutazioni inattivanti si associano a loss of function del complesso della succinato deidrogenasi, con conseguente accumulo del succinato a livello mitocondriale; quest'ultimo inibisce, a sua volta, l'enzima prolil-idrossilasi, che non risulta quindi in grado di idrossilare e inattivare il fattore di trascrizione ipossia-inducibile di tipo 1 alfa $(\mathrm{HIF} 1 \alpha)$. Di conseguenza, si verifica la trascrizione dei geni responsivi a HIF $1 \alpha$, in maniera analoga a ciò che avviene in uno stato ipossico [2].

La differente tempistica di esordio delle manifestazioni è imputabile al fatto che, nonostante la mutazione germinale avvenga a carico di un solo allele dei geni SDHx, è necessaria la delezione a livello somatico del secondo allele, con conseguente perdita dell'eterozigosi, per dar origine all'oncogenesi, in accordo con il modello two hits di Knudson [2].

Anche le mutazioni dei geni TMEM127 e MAX vengono trasmesse con ereditarietà autosomica dominante. Il gene TMEM127 codifica per una proteina transmembrana che riduce il signaling di mTOR; in caso di mutazione inattivante, mTOR risulta costituzionalmente attivato. Il gene MAX, infine, codifica per un fattore di trascrizione che regola l'espressione dell'oncogene Myc, modulando la crescita cellulare, la differenziazione e l'apoptosi. La sua variante mutata a livello germinale e la successiva perdita dell'eterozigosi a livello somatico portano alla disinibizione di Myc $[2,5,6]$.

\section{PPGL e adenomi ipofisari}

I tumori ipofisari e i PPGL sono patologie relativamente rare; nonostante ciò, talvolta si manifestano contemporaneamente nello stesso individuo o in una stessa famiglia. La prima descrizione di un caso di acromegalia e feocromoci- 
toma risale al 1952. La loro coesistenza può essere il frutto di una pura coincidenza, così come è possibile che, in alcuni casi, vi sia un meccanismo patogenetico comune alla base di entrambe le condizioni cliniche. In letteratura sono state formulate diverse spiegazioni per questo fenomeno: una mutazione di predisposizione ai PPGL può causare l'insorgenza di un PIT o, viceversa, una mutazione di predisposizione a PIT può determinare anche lo sviluppo di un PPGL. Inoltre, possono coesistere due mutazioni geniche differenti o può essere presente una mutazione a carico di un gene non noto responsabile di entrambe le patologie. Infine, la produzione ectopica di ormoni ipotalamo-ipofisari, quali ACTH e GH$\mathrm{RH}$, da parte di un PPGL potrebbe mimare la presenza di un PIT [7].

In particolare, vi sono evidenze crescenti secondo cui i geni di suscettibilità ai PPGL possano giocare un ruolo nella genesi tumorale ipofisaria [8, 9]. Un case report del 2012 ha evidenziato, per la prima volta, il potenziale coinvolgimento della mutazione SDHD nello sviluppo di un PIT. Si trattava di un soggetto di 37 anni, in presenza di familiarità per PPGL, con diagnosi di feocromocitoma bilaterale, macroadenoma GH-secernente e riscontro di mutazione a carico del gene SDHD. Il ruolo patogenetico ipofisario del suddetto difetto genetico è stato dimostrato mediante il riscontro di: 1) perdita dell'eterozigosi del locus genico SDHD a livello somatico nelle cellule dell'adenoma GHsecernente; e 2) mancata espressione all'analisi immunoistochimica (IC) della proteina SDHD nelle cellule neoplastiche ipofisarie [10]. Successivamente sono stati descritti ulteriori casi di PIT tra i pazienti con mutazioni SDHx. Pertanto, al giorno d'oggi, è globalmente accettato che l'associazione tra PIT, PCC e PGL, definita $3 P$ association syndrome (3PAs), rappresenti una nuova forma di predisposizione ereditaria a multiple neoplasie endocrine, correlata in prevalenza a mutazione dei geni SDHx [9].

La presenza di una mutazione SDHx nei soggetti con PIT è comunque un'evenienza rara e risulta più frequente in caso di concomitante PPGL o in presenza di anamnesi familiare positiva per PPGL [7]. Denes e colleghi hanno osservato una coorte di 39 pazienti con PPGL e/o PIT sporadici o familiari, di cui 19 individui con PPGL e PIT coesistenti e i restanti 20 con solo una delle due manifestazioni, in presenza tuttavia di storia familiare positiva per entrambe le neoplasie endocrine. Tra i PIT, i sottotipi più frequentemente riscontrati erano i prolattinomi, seguiti da adenomi GH-secernenti, non funzionanti e con IC positiva per LH/FSH. Tra i soggetti con evidenza contestuale di PPGL e PIT, 8 (42,1\%) presentavano una mutazione di uno dei geni SDHx (SDHB, SDHC, SDHD e una variante genetica di non chiaro significato patogenetico per SDHA e SDHAF2), di cui 5 nel contesto di famiglie con anamnesi positiva per PPGL. Da ultimo, nella serie di pazienti con PPGL e PIT, sono state riscontrate 2 mutazioni a carico del gene VHL e 2 a livello del gene menina; al contrario, non è stata identificata alcuna mutazione di
RET, TMEM127, MAX, FH, AIP e CDKN1B [8]. In letteratura non sono stati riportati finora altri casi di coesistenza di PIT e PPGL in soggetti con mutazione VHL. Pertanto, considerando la frequenza con cui i soggetti con sindrome di VHL eseguono esami neurologici di imaging, la bassa frequenza può indicare che l'associazione tra mutazione del gene VHL e PIT sia casuale, ovvero non geneticamente determinata [9]. Al contrario, nei due soggetti con mutazione di menina è stata riscontrata perdita dell'eterozigosi a livello somatico nelle cellule di PCC, a suggerire che i PPGL possono rappresentare potenziali manifestazioni della sindrome MEN1, come peraltro riscontrato in modelli murini knock-out per tale gene. Questo suggerisce l'indicazione a valutare l'esecuzione del test per la ricerca della mutazione di menina nei soggetti con diagnosi di PPGL, in presenza di altre caratteristiche cliniche suggestive per MEN1. Infine, dovrebbe essere preso in considerazione lo screening per la sindrome MEN4 (correlata a mutazione del gene CDKN1B) in soggetti con PIT e PPGL e fenotipo MEN1-simile, senza mutazione a carico di menina e SDHx [8].

Più recentemente, è stata condotta una revisione di tutti i case report presenti in letteratura di coesistenza di PPGL e PIT (dal 1952 al 2019) con identificazione di 82 casi, di cui 31 soggetti $(37,8 \%)$ presentavano una mutazione a carico di uno dei geni di suscettibilità a PPGL o PIT. Tra tutti i soggetti con 3PAs, 22 pazienti $(26,82 \%)$ avevano una storia personale o familiare indicativa di una sindrome endocrina ereditaria, mentre $37(45,1 \%)$ erano casi isolati e dei restanti 23 soggetti non si avevano a disposizione informazioni riguardanti le rispettive famiglie. In merito alla frequenza delle mutazioni geniche identificate, la maggior parte dei soggetti mutati presentava un'alterazione a carico dei geni SDHx (19 casi su 31), seguita in ordine decrescente da quella dei geni menina e MAX. Tuttavia, considerando la natura retrospettiva del lavoro e la mancata esecuzione del test genetico in una grossa proporzione di casi, soprattutto quelli meno recenti, non è possibile la generalizzazione del dato (Fig. 2) [9]. Malgrado l'identificazione di nuove mutazioni/varianti geniche associate alla 3PAs, tra cui quella del gene MAX, nella maggior parte dei casi non si è in grado di identificare il difetto genetico predisponente allo sviluppo di PPGL e PIT, in quanto verosimilmente associati a mutazioni non ancora riconosciute.

\section{Caratteristiche cliniche e istopatologiche degli adenomi ipofisari nel contesto della 3PAs}

Una caratteristica istopatologica che contraddistingue le cellule di un PIT correlato a mutazione SDHx è la presenza di vacuoli intracitoplasmatici [7, 8]. Dal punto di vista clinico i PIT, nel contesto di una 3PAs, sono rappresentati prevalentemente da prolattinomi, adenomi GH-secernenti o non funzionanti, presentano un comportamento aggressivo e so- 

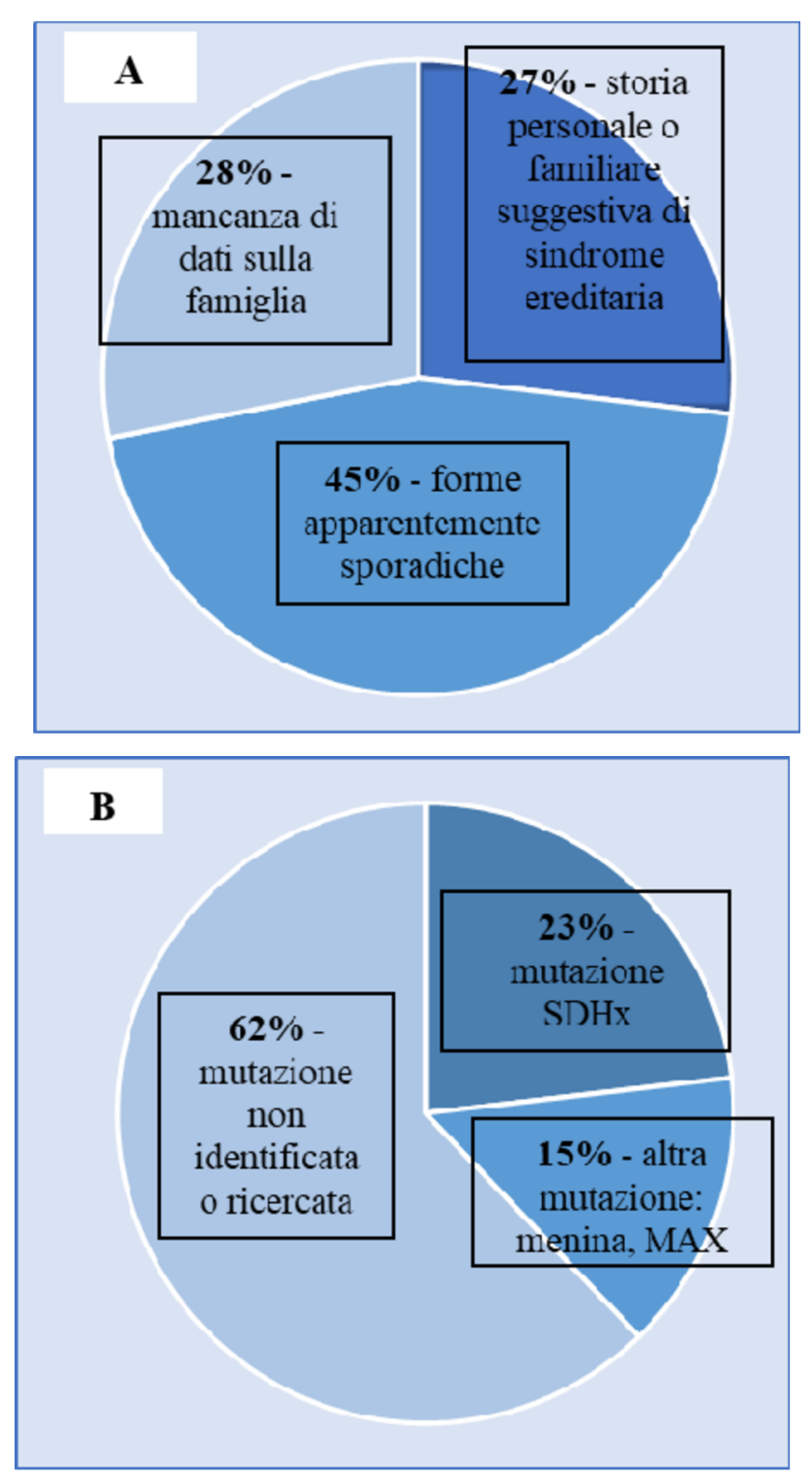

Fig. 2 Casi pubblicati in letteratura di coesistenza tra adenomi ipofisari e PPGL. Nel pannello a la popolazione è stata suddivisa sulla base della presentazione familiare, mentre nel pannello b sulla base della presenza o meno e del tipo di mutazione genetica identificata [9]

no più frequentemente resistenti al trattamento rispetto alle forme isolate. I PIT isolati associati a mutazione SDHx vengono diagnosticati a un'età più precoce rispetto ai casi con 3PAs senza evidenza di mutazione genetica. Al contrario, questi ultimi sono più spesso dei macroadenomi e necessitano di più linee terapeutiche, in quanto probabilmente la coesistenza del PPGL ne favorisce la crescita e la condizione di resistenza. Rispetto alle forme sporadiche, spesso, richiedono terapie multiple identificandosi come neoplasie più aggressive [11]. Non sembra esservi una correlazione genotipo-fenotipo, sebbene un esiguo numero di famiglie con 3PAs abbia sviluppato altre neoplasie correlate alle mutazioni SDHx, tra cui i GIST e gli RCC [9].

\section{Raccomandazioni per lo screening genetico e la} sorveglianza clinica nei soggetti con 3PAs

I soggetti con diagnosi contestuale di PPGL e PIT (i.e. 3PAs) e familiarità per PPGL, vengono sottoposti a test genetico per ricerca della mutazione a carico dei geni SDHx. Al contrario, in assenza di storia familiare positiva, ma in presenza di caratteristiche fenotipiche suggestive per MEN1/MEN2, si deve ricercare in prima battuta la mutazione a carico di menina o RET e altrimenti di SDHx, MAX o degli altri geni di suscettibilità ai PIT (AIP, CDKN1B).

Tutti i parenti di primo grado di soggetti affetti da 3PAs associata a mutazione SDHx devono essere sottoposti a test genetico; se viene identificata la mutazione, deve essere effettuata una valutazione clinica e biochimica basale per la ricerca di PPGL e di PIT (metanefrine frazionate plasmatiche e urinarie delle 24 ore e tropine ipofisarie) e in caso di positività devono essere eseguite indagini morfologiche di localizzazione. Al contrario, qualora non venga riscontrata alcuna alterazione, si pone indicazione a follow-up biochimico annuale (Fig. 3) [9].

\section{PPGL e RCC a cellule chiare}

L'associazione di PCC e RCC a cellule chiare è stata descritta per la prima volta nella sindrome di VHL oltre 60 anni fa. Nel 2017, Casey e colleghi hanno descritto una sindrome caratterizzata dall'insorgenza, in un singolo individuo o nei familiari di primo grado, di PPGL e RCC in assenza di mutazione germinale del gene VHL. Tale sindrome è stata definita Renal And PheochromocytomalParaganglioma Tumor Association Syndrome (RAPTAS). Dall'analisi di una casistica di 32 pazienti è emerso come la mutazione più frequentemente riscontrata sia a carico di SDHB, sebbene siano state evidenziate anche mutazioni di SDHC, SDHD, TMEM127 e MAX. È tuttavia opportuno sottolineare come, in una quota rilevante di pazienti con RAPTAS, non sia emersa alcuna delle suddette mutazioni, a indicare la probabile esistenza di geni di suscettibilità non ancora identificati. Gli autori hanno inoltre proposto un algoritmo diagnostico per guidare l'analisi genetica nei pazienti con RAPTAS, riassunto in Figura 4 [12].

\section{PPGL e GIST}

I GIST sono i più frequenti tumori mesenchimali del tratto gastro-intestinale. Originano dalle cellule interstiziali di Cajal, cellule "pacemaker" che regolano la motilità intestinale, localizzate prevalentemente a livello dello stomaco e del piccolo intestino.

Circa l' $85 \%$ dei GIST dell'adulto presenta una mutazione somatica a livello dei recettori tirosin-chinasici KIT o 


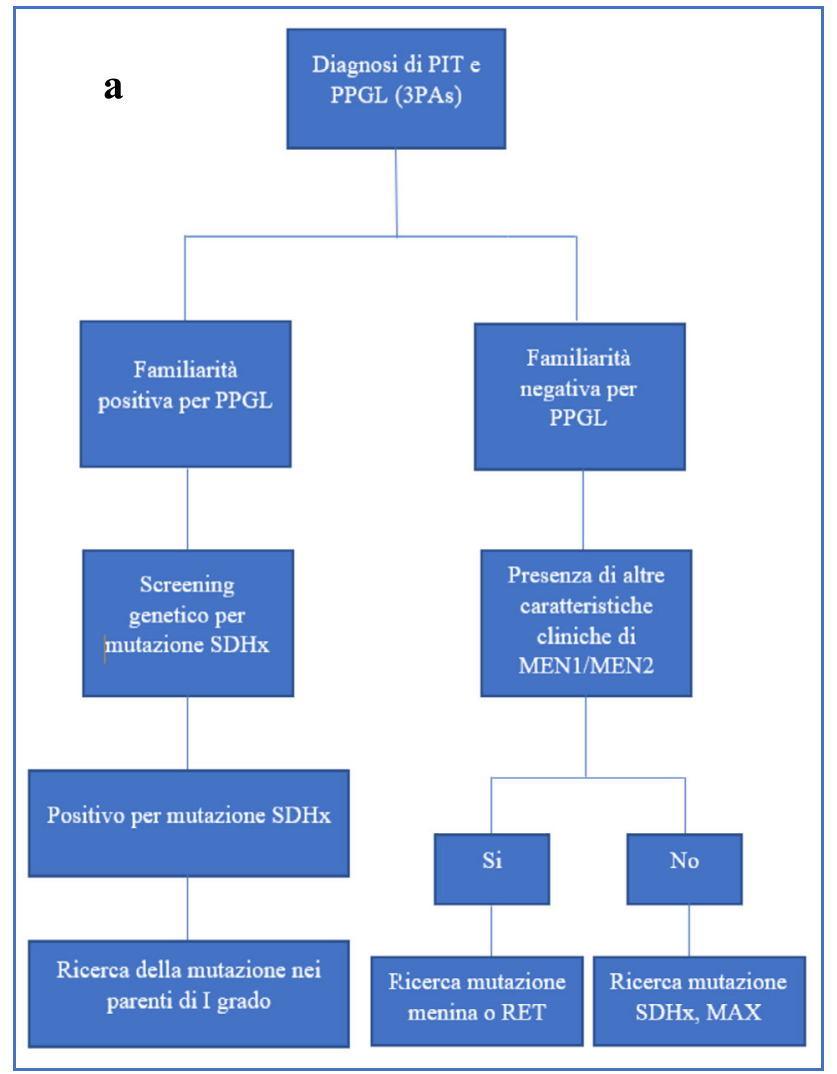

Fig. 3 Indicazioni per la sorveglianza clinica nei pazienti con diagnosi di 3PAs (a) e per lo screening genetico nei familiari (b) [9]. $3 P A s$, Sindrome dell'associazione delle $3 \mathrm{P} ; M E N$ 1, Sindrome da

PDGFRA. Il restante 10-15\% dei GIST, invece, non presenta mutazioni in questi due geni e viene pertanto definito GIST wild-type. Circa la metà dei GIST wild-type si associa a perdita di espressione di SDHB all'IC, mentre i GIST wild-type con conservata espressione di SDHB si associano a mutazioni dei geni BRAF e NF1.

I GIST con perdita di espressione di SDHB sono più frequenti in età pediatrica e nei giovani adulti, sono quasi invariabilmente a localizzazione gastrica e tendono ad essere multifocali, con istologia epitelioide e pattern di crescita plessiforme. Nonostante il decorso della neoplasia possa essere indolente, sono frequenti le metastasi a livello linfonodale ed epatico [13].

L'associazione di GIST con perdita di espressione di SDHB e PGL è stata descritta nella triade di Carney (Carney Triad, CT) e nella sindrome di Carney-Stratakis (Carney Stratakis Syndrome, CSS) [14, 15].

La CT fu descritta per la prima volta nel 1977 come l'associazione di leiomiosarcomi gastrici (rivelatisi in seguito GIST), PGL e condromi polmonari. In questi pazienti sono state inoltre descritte altre neoplasie, quali adenomi surrenalici e leiomiomi esofagei. Pur essendo caratterizzata da perdita di espressione di SDHB all'IC, la CT non è associa-

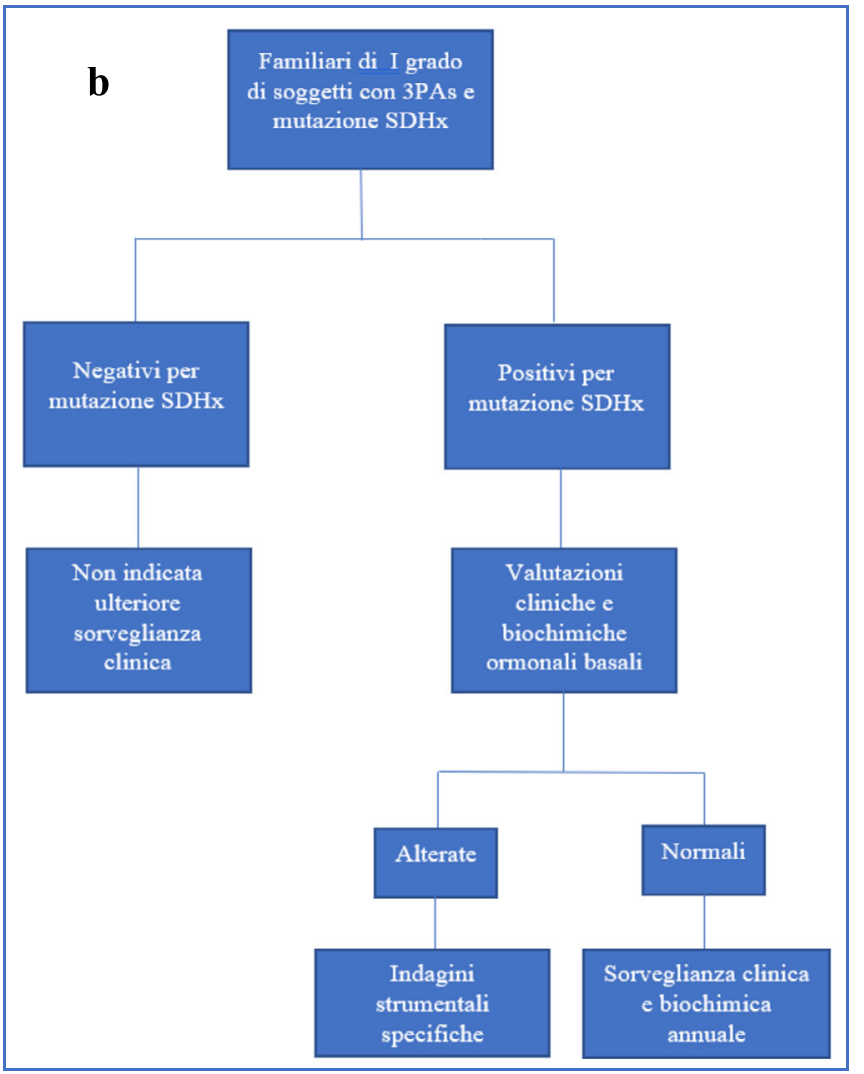

Neoplasie Endocrine Multiple di tipo 1; MEN 2, Sindrome da Neoplasie Endocrine Multiple di tipo 2; PIT, adenoma ipofisario; PPGL, feocromocitoma/paraganglioma

ta alla presenza di mutazioni germinali nei geni SDHx, ma sembra essere legata a meccanismi epigenetici. In particolare, è stato ipotizzato che l'ipermetilazione del promotore di SDHC ne provochi il silenziamento [16]. Nella quasi totalità dei casi, i soggetti affetti sono di sesso femminile, con esordio nella terza decade di vita. In uno studio su 37 pazienti con CT e PPGL, il 92\% presentava un paraganglioma extrasurrenalico, mentre il $16 \%$ un PCC. I paragangliomi erano multipli nel $22 \%$ dei casi, mentre il feocromocitoma era bilaterale nel $3 \%$ dei casi; nell' $11 \%$ dei casi si osservavano metastasi [15].

La CSS fu invece descritta per la prima volta nel 2002, come una condizione ereditaria a trasmissione autosomica dominante e penetranza incompleta caratterizzata dall'associazione di GIST gastrici e PPGL, causata da mutazioni germinali dei geni SDHx. La CSS sembra colpire in egual misura i due sessi, con esordio nella terza/quarta decade di vita; i PPGL sono nella maggior parte dei casi extra-surrenalici e multipli [13-15].

In conclusione, la presenza di GIST multifocali con istologia epitelioide ed esordio giovanile dovrebbe far sospettare una forma sindromica. In questi casi, è opportuna la valutazione dell'espressione di SDHB con IC e, in caso di per- 
Fig. 4 Algoritmo diagnostico in pazienti con RAPTAS [13]. RAPTAS, Renal and pheochromocytoma/paraganglioma tumor association syndrome; $P P G L$, feocromocitoma/paraganglioma; $R C C$, carcinoma a cellule renali; $S N C$, sistema nervoso centrale; $V H L$, Von Hippel Lindau

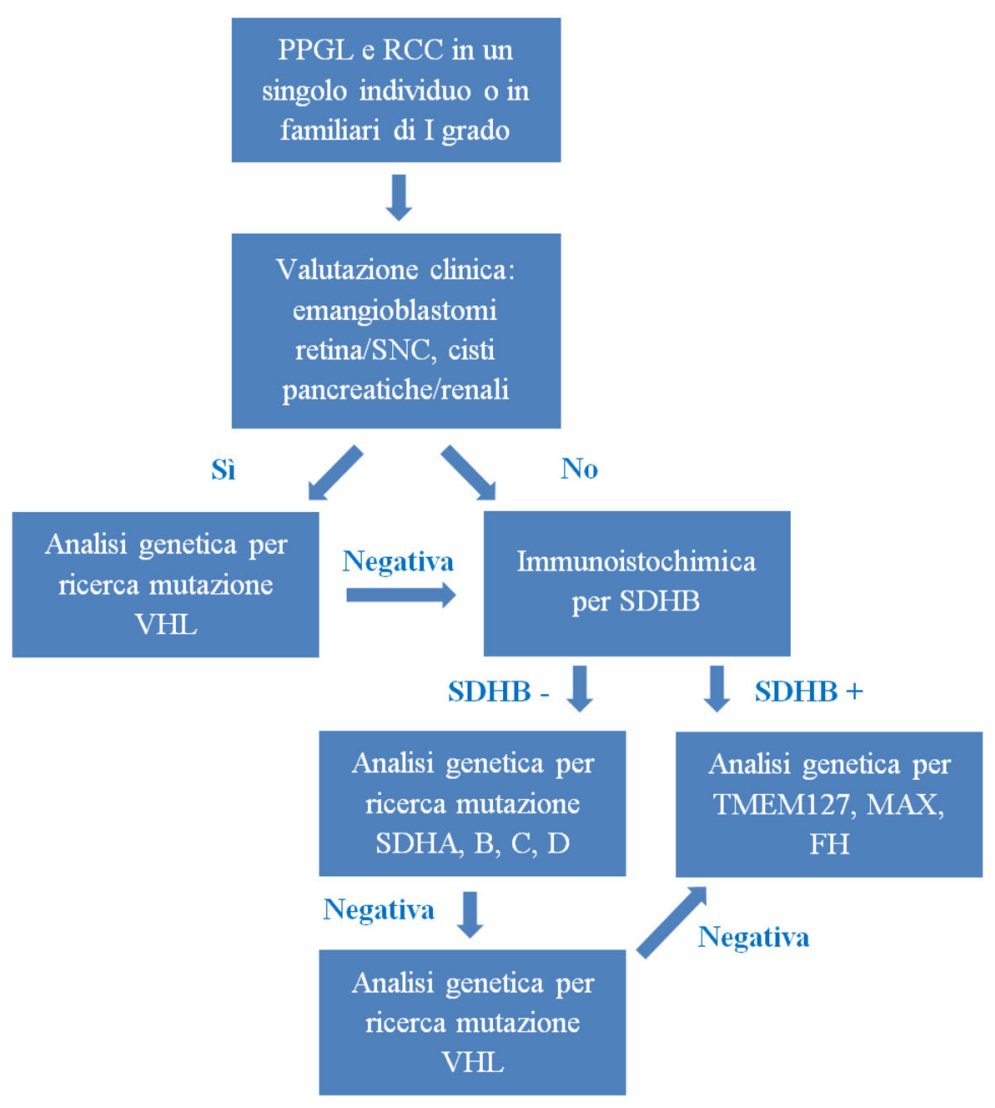

dita di espressione di tale proteina, viene richiesta l'analisi genetica. Il riscontro di una mutazione di SDHx, infatti, ha importanti implicazioni sulla successiva gestione clinica del paziente e dei familiari. Nei pazienti portatori di mutazione di SDHx, viceversa, non sembra essere raccomandato uno screening sistematico per la ricerca di GIST, in considerazione della prevalenza relativamente bassa di tali neoplasie in questi pazienti [14].

Feocromocitoma e GIST possono coesistere anche in pazienti affetti da NF1, sindrome neurocutanea causata dalla mutazione germinale del gene della neurofibromina e caratterizzata dalla presenza di alterazioni cutanee caratteristiche (macchie caffè-latte, lentiggini ascellari e inguinali, neurofibromi cutanei) e dalla predisposizione allo sviluppo di numerose neoplasie benigne e maligne. Sia nei feocromocitomi, sia nei GIST, è stato infatti dimostrato un possibile ruolo patogenetico dell'inattivazione del gene NF1.

I GIST rappresentano la più frequente manifestazione gastrointestinale nei pazienti con NF1, interessando il 7-25\% dei soggetti affetti da tale sindrome. I GIST associati a NF1 insorgono in genere in età adulta, sono multinodulari/multifocali e tipicamente si localizzano a livello del piccolo intestino; risultano wild-type per mutazioni di KIT e PDGFRA e presentano conservata espressione di SDHB all'IC [13].

L'1-5\% dei pazienti con NF1 sviluppa un PPGL, con un'età media alla diagnosi di 42 anni. Nel 95\% dei casi si tratta di PCC secernenti, unilaterali e a comportamento biologico benigno, con pattern secretorio prevalentemente adrenergico [15].

L'associazione di PPGL e GIST in pazienti affetti da NF1 sembra comunque molto rara. Nei case report, l'età alla diagnosi è superiore a 40 anni, i PPGL presentano localizzazione surrenalica unilaterale o bilaterale e, in alcuni casi, sono parte di un tumore composito (feocromocitoma/ganglioneuroma, feocromocitoma/tumore maligno delle guaine nervose periferiche); i GIST sono in genere multinodulari/multifocali e localizzati prevalentemente a livello del piccolo intestino $[17,18]$.

\section{Conclusioni}

Le mutazioni di predisposizione ai PPGL identificate più recentemente, tra cui in particolare quelle dei geni SDHx, possono favorire, seppur con bassa frequenza, anche l'insorgenza di altre neoplasie: GIST, RCC e PIT [2, 5]. Pertanto, nei soggetti con diagnosi di GIST, RCC e PIT il clinico dovrebbe valutare attentamente l'anamnesi personale e familiare alla ricerca di eventuali PPGL associati: in presenza di fenotipo clinico/patologico suggestivo di forma ereditaria viene proposta la ricerca in IC del prodotto genico a livello del tessuto neoplastico e il successivo test genetico in caso di 
mancata espressione della proteina specifica. Dall'altro lato, i pazienti con diagnosi di PPGL associato a mutazione di SDHx, TMEM127 e MAX dovrebbero essere accuratamente indagati per la ricerca di eventuali segni e sintomi di neoplasie potenzialmente correlate. Al momento attuale, tuttavia, non è raccomandata una ricerca sistematica di suddette manifestazioni, almeno fino a quando non saranno condotte indagini prospettiche volte a stabilirne la cadenza necessaria [14].

Funding Note Open access funding provided by Università degli Studi di Torino within the CRUI-CARE Agreement.

Conflitto di interesse Gli autori Chiara Lopez, Martina Bollati, Mirko Parasiliti-Caprino, Nunzia Prencipe, Alessandro Maria Berton, Ezio Ghigo, Silvia Grottoli e Mauro Maccario dichiarano di non avere conflitti di interesse. Silvia Grottoli fa parte del Comitato di redazione.

Consenso informato Lo studio presentato in questo articolo non ha richiesto sperimentazione umana.

Studi sugli animali Gli autori di questo articolo non hanno eseguito studi sugli animali.

Nota della casa editrice Springer Nature rimane neutrale in riguardo alle rivendicazioni giurisdizionali nelle mappe pubblicate e nelle affiliazioni istituzionali.

Open Access This article is licensed under a Creative Commons Attribution 4.0 International License, which permits use, sharing, adaptation, distribution and reproduction in any medium or format, as long as you give appropriate credit to the original author(s) and the source, provide a link to the Creative Commons licence, and indicate if changes were made. The images or other third party material in this article are included in the article's Creative Commons licence, unless indicated otherwise in a credit line to the material. If material is not included in the article's Creative Commons licence and your intended use is not permitted by statutory regulation or exceeds the permitted use, you will need to obtain permission directly from the copyright holder. To view a copy of this licence, visit http://creativecommons.org/licenses/by/4.0/.

\section{Bibliografia}

1. Lopez C, Egalini F, Parasiliti Caprino M et al (2020) Il versante endocrinologico della sindrome di von Hippel-Lindau (vHLs). Endocrinologo 21:332-337

2. Muth A, Crona J, Gimm O et al (2019) Genetic testing and surveillance guidelines in hereditary pheochromocytoma and paraganglioma. J Intern Med 285:187-204
3. Lenders JW, Duh QY, Eisenhofer G et al (2014) Pheochromocytoma and paraganglioma: an Endocrine Society clinical practice guideline. J Clin Endocrinol Metab 99:1915-1942

4. Fisbein L, Merril S, Fraker DL et al (2013) Inherited mutations in pheochromocytoma and paraganglioma: why all patients should be offered genetic testing. Ann Surg Oncol 20(5):1444-1450

5. Qui Y, Deng Y, Ricketts CJ et al (2014) The tumor susceptibility gene TMEM127 is mutated in renal cell carcinomas and modulates endolysosomal function. Hum Mol Genet 23(9):2428-2439

6. Korpershoek E, Koffy D, Eussen BH et al (2016) Complex MAX rearrangement in a family with malignant pheochromocytoma, renal oncocytoma and erythrocytosis. J Clin Endocrinol Metab 101(2):453-460

7. O'Toole SM, Denes J, Robledo M et al (2015) 15 Years of paraganglioma. The association of pituitary adenomas and phaeochromocytomas or paragangliomas. Endocr-Relat Cancer 22:T105-T122

8. Denes J, Swords F, Rattenberry E et al (2015) Heterogeneous genetic background of the association of pheochromocytoma/paraganglioma and pituitary adenoma: results from a large patient cohort. J Clin Endocrinol Metab 100(3):E531-E541

9. Xekouki P, Brennand A, Whitelaw B et al (2019) The 3PAs: an update on the association of pheochromocytomas, paragangliomas, and pituitary tumors. Horm Metab Res 51:419-436

10. Xekouki P, Pacak K, Almeida M et al (2012) Succinate dehydrogenase (SDH) D subunit (SDHD) inactivation in a growth-hormoneproducing pituitary tumor: a new association for SDH? J Clin Endocrinol Metab 97:E357-E366

11. Tatsi C, Stratakis C (2020) The genetics of pituitary adenomas. J Clin Med 9(1):30

12. Casey RT, Warren AY, Martin JE et al (2017) Clinical and molecular features of Renal and Pheochromocytoma/Paraganglioma Tumor Association Syndrome (RAPTAS): case series and literature review. J Clin Endocrinol Metab 102(11):4013-4022

13. Boikos SA, Stratakis CA (2014) The genetic landscape of gastrointestinal stromal tumor lacking KIT and PDGFRA mutations. Endocrine 47(2):401-408

14. Mannelli M, Canu L, Ercolino T et al (2018) Diagnosis of endocrine disease: SDHx mutations: beyond pheochromocytomas and paragangliomas. Eur J Endocrinol 178:R11-R17

15. Welander J, Söderkvist P, Gimm O (2011) Genetics and clinical characteristics of hereditary pheochromocytomas and paragangliomas. Endocr-Relat Cancer 18(6):R253-276

16. Settas N, Faucz FR, Stratakis CA (2018) Succinate dehydrogenase $(\mathrm{SDH})$ deficiency, Carney triad and the epigenome. Mol Cell Endocrinol 469:107-111

17. Vlenterie M, Flucke U, Hofbauer LC et al (2013) Pheochromocytoma and gastrointestinal stromal tumors in patients with Neurofibromatosis Type I. Am J Med 126:174-180

18. Gorgel A, Cetinkaya DD, Salgur F et al (2014) Coexistence of Gastrointestinal Stromal Tumors (GISTs) and pheochromocytoma in three cases of Neurofibromatosis type 1 (NF1) with a review of the literature. Intern Med 53:1783-1789 